\title{
Consensus building: How to persuade a group
}

\author{
Bernard Caillaud and Jean Tirole
}

May 7, 2007

\section{Additional materials}

\section{Proof of Lemma C1}

Referring to Appendix $\mathrm{C}$ and using feasibility constraints, note that a mechanism is alternatively given by $\left(\gamma, \theta_{i}, \mu_{i}, \bar{\mu}_{i}, \nu_{0}, \nu_{3}\right)$ and:

$$
\begin{aligned}
\eta & =1-\gamma-\bar{\mu}_{1}-\bar{\mu}_{2}-\nu_{0} \geq 0, \\
\nu_{1} & =\nu_{0}-\theta_{1}-\mu_{1}+\bar{\mu}_{1} \geq 0, \\
\nu_{2} & =\nu_{0}-\theta_{2}-\mu_{2}+\bar{\mu}_{2} \geq 0, \\
\xi_{3} & =\nu_{0}-\theta_{1}-\theta_{2}-\mu_{1}-\mu_{2}+\bar{\mu}_{1}+\bar{\mu}_{2}-\nu_{3} \geq 0 .
\end{aligned}
$$

Using measurability and individual rationality, the expected probability that the project is implemented is given by:

$$
Q=\gamma+p_{1} \theta_{1}+p_{2} \theta_{2}+P \xi_{3}
$$

Plugging in the value of $\xi_{3}$ from (4), we find:

$$
Q=\gamma+\left(p_{1}-P\right) \theta_{1}+\left(p_{2}-P\right) \theta_{2}+P \nu_{0}-P \mu_{1}-P \mu_{2}+P \bar{\mu}_{1}+P \bar{\mu}_{2}-P \nu_{3}
$$


We now write incentive constraints using measurability, individual rationality and feasibility constraints. Equation (C1) in Appendix C can be written as:

$$
\begin{aligned}
& \theta_{i} p_{i}(G-c)-\left(\mu_{i} p_{i}+\bar{\mu}_{i}\left(1-p_{i}\right)\right) c+\xi_{3} P(G-c) \\
& -\left(\nu_{0}\left(1+P-p_{1}-p_{2}\right)+\nu_{1}\left(p_{1}-P\right)+\nu_{2}\left(p_{2}-P\right)+\nu_{3} P\right) c \\
\geq & \theta_{i} p_{i} G-\theta_{i}\left(1-p_{i}\right) L+\xi_{3} P G-\xi_{3}\left(p_{j}-P\right) L .
\end{aligned}
$$

Given previous results and using the expressions for $\xi_{3}$ and $\nu_{i}$, this constraint can be written as: for $i=1,2$ and $j \neq i$,

$$
\begin{aligned}
& \theta_{i}\left(1-p_{i}\right) L+\left(\nu_{0}-\theta_{1}-\theta_{2}-\mu_{1}-\mu_{2}+\bar{\mu}_{1}+\bar{\mu}_{2}-\nu_{3}\right)\left(p_{j}-P\right) L \\
\geq & c\left[\nu_{0}+\bar{\mu}_{i}+p_{j}\left(\bar{\mu}_{j}-\theta_{j}-\mu_{j}\right)\right] .
\end{aligned}
$$

Equation (C2) in Appendix $\mathrm{C}$ can be written as:

$$
\begin{aligned}
0 \leq & \theta_{i} p_{i}(G-c)-\left(\mu_{i} p_{i}+\bar{\mu}_{i}\left(1-p_{i}\right)\right) c+\xi_{3} P(G-c) \\
& -\left(\nu_{0}\left(1+P-p_{1}-p_{2}\right)+\nu_{1}\left(p_{1}-P\right)+\nu_{2}\left(p_{2}-P\right)+\nu_{3} P\right) c .
\end{aligned}
$$

Using the same manipulations as above, the latter inequality becomes: for $i=1,2$ and $j \neq i$,

$$
\begin{aligned}
& \theta_{i} p_{i} G+\left(\nu_{0}-\theta_{1}-\theta_{2}-\mu_{1}-\mu_{2}+\bar{\mu}_{1}+\bar{\mu}_{2}-\nu_{3}\right) P G \\
\geq & c\left[\nu_{0}+\bar{\mu}_{i}+p_{j}\left(\bar{\mu}_{j}-\theta_{j}-\mu_{j}\right)\right] .
\end{aligned}
$$

Finally, we write Equation (C3) in Appendix C as follows: for $i=1,2$ and $j \neq i$,

$$
\gamma u^{R}\left(p_{i}\right)+\theta_{j} p_{j} u^{R}\left(\hat{p}_{i}\right) \geq 0 \text {. }
$$

The program is to maximize (5) under the constraints (1)-(2)-(3)-(4), (7), (9) and (10). 
It is first immediate that $\nu_{3}=0$ at the optimum. With $A_{i}, B_{i}$ and $C_{i}$ the multipliers associated with constraints (7), (9) and (10), and $D, E_{1}, E_{2}$ and $F$ the multipliers associated with (1)-(2)-(3)-(4), one can compute the derivatives of the Lagrangian with respect to $\left(\mu_{1}, \mu_{2}, \bar{\mu}_{1}, \bar{\mu}_{2}, \nu_{0}\right)$ (omitting the constraints that each of these must lie within $\left.[0,1]\right)$ :

$$
\begin{gathered}
\frac{\partial \mathcal{L}}{\partial \mu_{i}}=-P-A_{i}\left(p_{j}-P\right) L-A_{j}\left(p_{i}-P\right) L+A_{j} c p_{i} \\
-B_{i} P G-B_{j} P G+B_{j} c p_{i}-\left(D+E_{i}\right) \\
\frac{\partial \mathcal{L}}{\partial \bar{\mu}_{i}}=\quad P+A_{i}\left(p_{j}-P\right) L+A_{j}\left(p_{i}-P\right) L-A_{j} c p_{i} \\
+B_{i} P G+B_{j} P G-B_{j} c p_{i}-c\left(A_{i}+B_{i}\right)+\left(D+E_{i}\right)-F \\
\frac{\partial \mathcal{L}}{\partial \nu_{0}}=\quad P+A_{1}\left(p_{2}-P\right) L-c A_{1}+A_{2}\left(p_{1}-P\right) L-c A_{2} \\
+B_{1} P G-c B_{1}+B_{2} P G-c B_{2}+\left(D+E_{1}+E_{2}\right)-F
\end{gathered}
$$

Note that if $\left(A_{j}+B_{j}\right)=0$, then $\frac{\partial \mathcal{L}}{\partial \mu_{i}}<0$ and so, $\mu_{i}=0$.

From the derivatives of the Lagrangian, one can derive useful relationships:

$$
\begin{gathered}
\frac{\partial \mathcal{L}}{\partial \mu_{i}}+\frac{\partial \mathcal{L}}{\partial \bar{\mu}_{i}}=-F-c\left(A_{i}+B_{i}\right) \leq 0 \\
\frac{\partial \mathcal{L}}{\partial \bar{\mu}_{i}}+E_{j}=\frac{\partial \mathcal{L}}{\partial \nu_{0}}+c\left(1-p_{i}\right)\left(A_{j}+B_{j}\right) .
\end{gathered}
$$

Claim 1. The optimum cannot be such that $\nu_{0}>0, \mu_{1}>0$ and $\mu_{2}>0$.

Proof: If $\nu_{0}>0, \mu_{i}>0$ for $i=1,2$, it follows that $\frac{\partial \mathcal{L}}{\partial \nu_{0}} \geq 0, \frac{\partial \mathcal{L}}{\partial \mu_{i}} \geq 0 . A_{1}, A_{2}, B_{1}$ and $B_{2}$ must be strictly positive so that $\frac{\partial \mathcal{L}}{\partial \mu_{i}}+\frac{\partial \mathcal{L}}{\partial \bar{\mu}_{i}}<0$. Hence, $\frac{\partial \mathcal{L}}{\partial \bar{\mu}_{i}}<0$ and $\bar{\mu}_{i}=0$ from (11).

Moreover, (12) implies that $E_{j}>0$, which implies $\nu_{j}=0$ and so, summing (2) and $(3), \xi_{3}=-\nu_{0}<0$, a contradiction.

Claim 2. The optimum is without loss of generality such that for $i=1,2, \mu_{i} \bar{\mu}_{i}=0$. 
Proof: Fix $\bar{\mu}_{i}-\mu_{i}$. A simple examination of $Q$ and of all the constraints reveals that decreasing $\bar{\mu}_{i}$ only relaxes (1) and (7)-(9). Therefore, if $\bar{\mu}_{i}-\mu_{i} \geq 0$, the optimum can be chosen so that $\mu_{i}=0$ and if $\bar{\mu}_{i}-\mu_{i} \leq 0$, the optimum can be chosen so that $\bar{\mu}_{i}=0$.

Therefore, we will now focus on optima that satisfy Claim 2.

Claim 3. An optimum satisfying Claim 2 cannot be such that $\nu_{0}=0$ and $\mu_{i}>0$ for some $i$.

Proof: Suppose that $\nu_{0}=0$ and there exists $i$ such that $\mu_{i}>0$. From Claim 2, the optimum is such that $\bar{\mu}_{i}=0$. Then, the constraint that $\nu_{i} \geq 0$ is violated.

Claim 4. An optimum satisfying Claim 2 cannot be such that $\nu_{0}>0, \mu_{1}>0$ and $\mu_{2}=0$.

Proof: Suppose $\nu_{0}>0$ and $\mu_{1}>0=\mu_{2}=\bar{\mu}_{1}$. It must be that $\frac{\partial \mathcal{L}}{\partial \nu_{0}} \geq 0, \frac{\partial \mathcal{L}}{\partial \mu_{1}} \geq 0, \frac{\partial \mathcal{L}}{\partial \bar{\mu}_{1}} \leq 0$ and $A_{2}+B_{2}>0$. As in the proof of Claim 1, it follows that $E_{2}>0$, which implies that $\nu_{2}=0$. So, we have:

$$
\begin{aligned}
0 & \leq \xi_{3}=\nu_{0}-\theta_{1}-\theta_{2}-\mu_{1}-\mu_{2}+\bar{\mu}_{1}+\bar{\mu}_{2} \\
& =\nu_{2}-\theta_{1}-\mu_{1}+\bar{\mu}_{1}=-\theta_{1}-\mu_{1}<0,
\end{aligned}
$$

a contradiction.

Claim 5. If $\mu_{1}=\mu_{2}=0$, the optimum is without loss of generality such that $\nu_{0}=0$.

Proof: Suppose $\mu_{1}=\mu_{2}=0<\nu_{0}$, then $\frac{\partial \mathcal{L}}{\partial \nu_{0}} \geq 0$.

Note first that if there exists $i$ such that $\frac{\partial \mathcal{L}}{\partial \bar{\mu}_{i}}>0$, then $\bar{\mu}_{i}=1$ and then $\eta<0$, a contradiction. So, for $i=1,2, \frac{\partial \mathcal{L}}{\partial \bar{\mu}_{i}} \leq 0$.

Note also that if $E_{i}>0$, then $\nu_{i}=0$ so that $\nu_{j}=\xi_{3}+\nu_{0}>0$ and therefore $E_{i}=0$. With the previous remark, using (12), this implies that $\frac{\partial \mathcal{L}}{\partial \nu_{0}}=0$ and for some $i, A_{i}=B_{i}=0$. 
Suppose $A_{1}=B_{1}=0<A_{2}+B_{2}$ and $E_{2}>0=E_{1}$. Consider the simplified program where the constraints corresponding to $A_{1}, B_{1}$ and $E_{1}$ are omitted. In this program, $\nu_{0}$ and $\bar{\mu}_{2}$ enter only through $\left(\nu_{0}+\bar{\mu}_{2}\right)$ within $(0,1]$; and so, there is no loss of generality in looking for the optimum with $\nu_{0}=0$.

The last possibility is such that $A_{i}=B_{i}=E_{i}=0$ for $i=1,2$. Then, the simplified program where all corresponding constraints are omitted only depends upon $\nu_{0}+\bar{\mu}_{1}+\bar{\mu}_{2}$, and again, one can set $\nu_{0}=0$ without loss of generality.

To summarize, the optimal mechanism is without loss of generality such that $\nu_{0}=$ $\mu_{1}=\mu_{2}=0$. It is fully characterized by $\left(\gamma, \theta_{1}, \theta_{2}, \bar{\mu}_{1}, \bar{\mu}_{2}\right)$, or, defining $\lambda_{i}=\bar{\mu}_{i}-\theta_{i}$, as in Lemma C1. This completes the proof of Lemma C1.

\section{Proof of Proposition C2}

In the symmetric setting, feasibility requires: $\gamma+\theta_{1}+\theta_{2}+\lambda_{1}+\lambda_{2}=1$. Incentive constraints (7), (9) and (10) now become:

$$
\begin{gathered}
\theta_{i}(1-p) L+\left(\lambda_{1}+\lambda_{2}\right)(p-P) L \geq c\left[\theta_{i}+\lambda_{i}+\lambda_{j} p\right], \\
\theta_{i} p G+\left(\lambda_{1}+\lambda_{2}\right) P G \geq c\left[\theta_{i}+\lambda_{i}+\lambda_{j} p\right], \\
\gamma(p G-(1-p) L)+\theta_{i} p(\hat{p} G-(1-\hat{p}) L) \geq 0 .
\end{gathered}
$$

The sponsor maximizes $Q=\gamma+\left(\theta_{1}+\theta_{2}\right) p+\left(\lambda_{1}+\lambda_{2}\right) P$ subject to these constraints.

If $\left(\gamma, \theta_{1}, \theta_{2}, \lambda_{1}, \lambda_{2}\right)$ is an optimal mechanism, $\left(\gamma, \frac{\theta_{1}+\theta_{2}}{2}, \frac{\theta_{1}+\theta_{2}}{2}, \frac{\lambda_{1}+\lambda_{2}}{2}, \frac{\lambda_{1}+\lambda_{2}}{2}\right)$ is a symmetric mechanism that satisfies the feasibility constraints, the incentive constraints, obtained by summing over $i=1$ and 2 the constraints (13), (14) and (15), and that yields the same $Q$. We will therefore focus wlog on symmetric mechanisms. 
For a symmetric mechanism $(\gamma, \theta, \lambda)$, feasibility requires $\gamma+2 \theta+2 \lambda=1$ and incentive constraints become:

$$
\begin{gathered}
\theta\left(p_{+}-p\right)+\lambda\left\{p_{+}(1+p)-(1-p)-2 P\right\} \geq 0 \\
\theta\left(p-p_{-}\right)+\lambda\left(2 P-(1+p) p_{-}\right) \geq 0 \\
\gamma\left(p-p_{0}\right)+\theta p\left(\hat{p}-p_{0}\right) \geq 0
\end{gathered}
$$

The sponsor maximizes $Q=\gamma+2 \theta p+2 \lambda P$ subject to these constraints. Since for $p \geq p_{0}$, the unconstrained optimum $(\gamma=1)$ is implementable, we focus on the case where $p<p_{0}$.

First case: $\hat{p}<p_{0}$. (18) implies that $\gamma=\theta=0$. The situation is the symmetric stochastic version of the deterministic situation in which both committee members investigate sequentially. If $P \geq p_{-}$, the optimum is $\lambda=\frac{1}{2}$ and $Q=P$, while $Q=0$ if $P<p_{-}$.

Second case: $p_{-} \leq p<p_{0}<\hat{p}$. Consider the relaxed program where (16) and (17) are omitted:

$$
\begin{aligned}
& \max _{\theta, \lambda \geq 0}\{-2 \theta(1-p)-2 \lambda(1-P)\} \\
\text { s.t. } 0 \leq & 1-2 \theta-2 \lambda \\
1 \leq & 2 \lambda+\theta \frac{\left[2\left(p_{0}-p\right)+p\left(\hat{p}-p_{0}\right)\right]}{p_{0}-p} .
\end{aligned}
$$

It is immediate that the solution is $\lambda=0$ and $\theta=\frac{1-\gamma}{2}=\theta^{*} \equiv \frac{p_{0}-p}{2\left(p_{0}-p\right)+p\left(\hat{p}-p_{0}\right)}$. Moreover, since $p-p_{-} \geq 0$ and $p_{+}-p>0$, this solution satisfies also (16) and (17). Hence, it is the optimal mechanism in this range of parameters.

Third case: $p<p_{-}<p_{0} \leq \hat{p}$. As in the previous case, we use variables $(\theta, \lambda) \geq 0$ such that $\gamma=1-2 \theta-2 \lambda \geq 0$. The constraints can be written as follows:

$$
\lambda \frac{\left[(1-p)+2 P-p_{+}(1+p)\right]}{p_{+}-p} \equiv X \lambda \leq \theta,
$$




$$
\theta \leq \lambda \frac{\left(2 P-(1+p) p_{-}\right)}{p_{-}-p} \equiv Y \lambda
$$

$$
1 \leq 2 \lambda+\frac{\theta}{\theta^{*}}
$$

Note first that if $Y \leq 0$, then $\theta=\lambda=0$ necessarily and the set of constraints is empty. Hence $Q=0$. Suppose now that $Y>0$. Again, if $X>Y$, then the set of constraints is empty and $Q=0$. The project can then be implemented with positive probability only if $Y \geq X$ and $Y>0$. In this last case, consider the relaxed program where the sole constraints are $\theta \geq 0, \lambda \geq 0,(20)$ and (21):

$$
\begin{aligned}
& \max _{\theta, \lambda \geq 0}\{-2 \theta(1-p)-2 \lambda(1-P)\} \\
\text { s.t. } \theta \leq & Y \lambda \\
1 \leq & 2 \lambda+\frac{\theta}{\theta^{*}} .
\end{aligned}
$$

The constraint (21) must necessarily be binding, since otherwise the optimum would be $\theta=\lambda=0$ which would violate (21). The constraint (20) must also be binding, since otherwise, the optimum would be $\lambda=0, \theta=\theta^{*}$ and this would violate (20). Hence, the solution is: $\theta=Y \lambda=\theta^{* *} \equiv\left(\frac{2}{Y}+\frac{1}{\theta^{*}}\right)^{-1}$. Moreover, since (20) is binding and $Y \geq X$, (19) is satisfied. For these values,

$$
\gamma=1-2 \theta^{* *}\left(1+\frac{1}{Y}\right)=\frac{\frac{1}{Y}+\frac{1}{\theta^{*}}-1}{\frac{2}{Y}+\frac{1}{\theta^{*}}} ;
$$

since $\theta^{*} \leq \frac{1}{2}$ and $Y>0, \gamma>0$.

Therefore, in the range $p<p_{-}<p_{0} \leq \hat{p}$, there exists a stochastic mechanism that yields a positive probability $Q$ if and only if:

$$
\begin{aligned}
& 2 P-(1+p) p_{-}>0 \text { and } \\
& \frac{2 P-(1+p) p_{-}}{p_{-}-p} \geq \frac{(1-p)+2 P-p_{+}(1+p)}{p_{+}-p}
\end{aligned}
$$


that is, if and only if:

$$
\begin{aligned}
& 2 P>(1+p) p_{-} \text {and } \\
& 2 P \geq(1+p) p+(1-p) \frac{p_{-}-p}{p_{+}-p_{-}} .
\end{aligned}
$$

The condition for $Q>0$ is therefore:

$$
\hat{p} \geq \max \left\{\frac{(1+p) p_{-}}{2 p}, \frac{(1+p)}{2}+\frac{(1-p)\left(p_{-}-p\right)}{2 p\left(p_{+}-p_{-}\right)}\right\} .
$$

In a left neighborhood of $p_{-}$, both terms in the supremum tends to $\frac{1+p_{-}}{2}<1$; therefore, the domain for which $Q>0$ is not empty. 